\title{
Characterizing the function of domain linkers in regulating the dynamics of multi-specific biologics by microsecond molecular dynamics simulations and artificial intelligence
}

\author{
Bo Wang ${ }^{1}$, Zhaoqian $\mathrm{Su}^{1}$, and Yinghao $\mathrm{Wu}^{1}$ \\ ${ }^{1}$ Albert Einstein College of Medicine
}

October 22, 2020

\begin{abstract}
Multi-domain proteins are not only formed through natural evolution but can also be generated by recombinant DNA technology. Because many fusion proteins can enhance the selectivity of cell targeting, these artificially produced molecules, called multispecific biologics, are promising drug candidates, especially for immunotherapy. Moreover, the rational design of domain linkers in fusion proteins is becoming an essential step toward a quantitative understanding of the dynamics in these biopharmaceutics. We developed a computational framework to characterize the impacts of peptide linkers on the dynamics of multi-specific biologics. We constructed a benchmark containing six types of linkers that represent various lengths and degrees of flexibility and used them to connect two natural proteins as a test system. The microsecond dynamics of these proteins generated from Anton were projected onto a coarse-grained conformational space. The similarity of dynamics among different proteins in this low-dimensional space was further analyzed by a neural network model. Finally, hierarchical clustering was applied to place linkers into different subgroups based on the neural network classification results. The clustering results suggest that the length of linkers used to spatially separate different functional modules plays the most important role in regulating the dynamics of this fusion protein. Given the same number of amino acids, linker flexibility functions as a regulator of protein dynamics. In summary, we illustrated that a new computational strategy can be used to study the dynamics of multi-domain fusion proteins by a combination of long timescale molecular dynamics simulation, coarse-grained modeling, and artificial intelligence.
\end{abstract}

\section{Hosted file}

AntonCGNN_Proofread10092020_clean.pdf available at https://authorea.com/users/369325/ articles/488229-characterizing-the-function-of-domain-linkers-in-regulating-thedynamics-of-multi-specific-biologics-by-microsecond-molecular-dynamics-simulationsand-artificial-intelligence 
a)
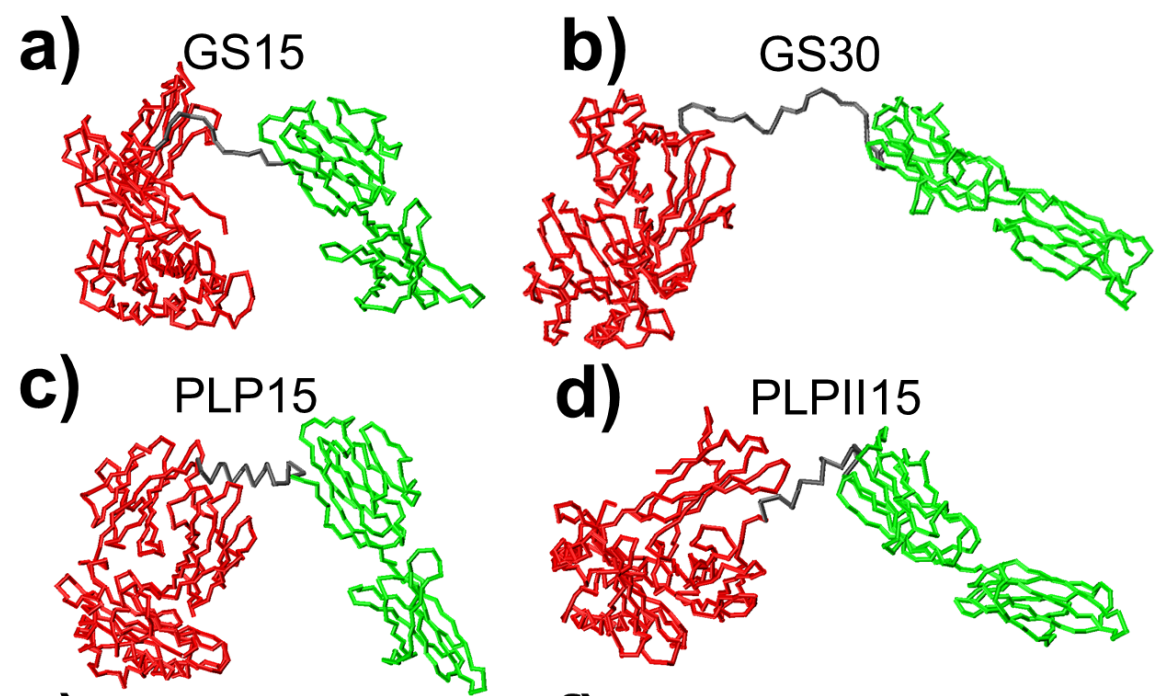

e) PLPII30
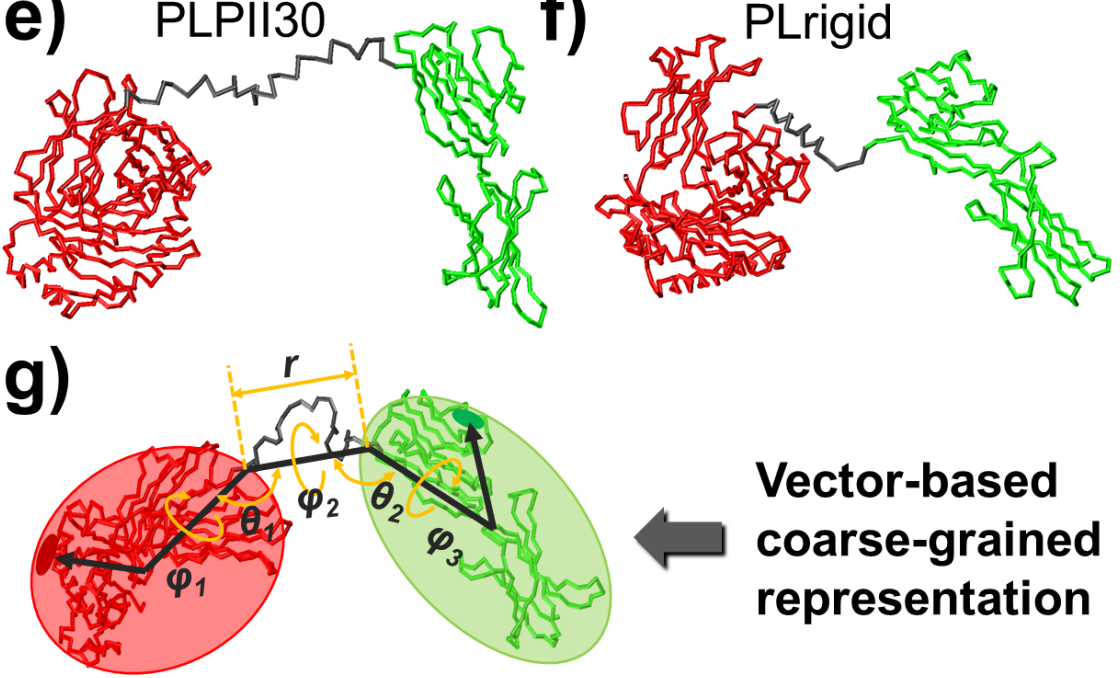

Vector-based coarse-grained representation 


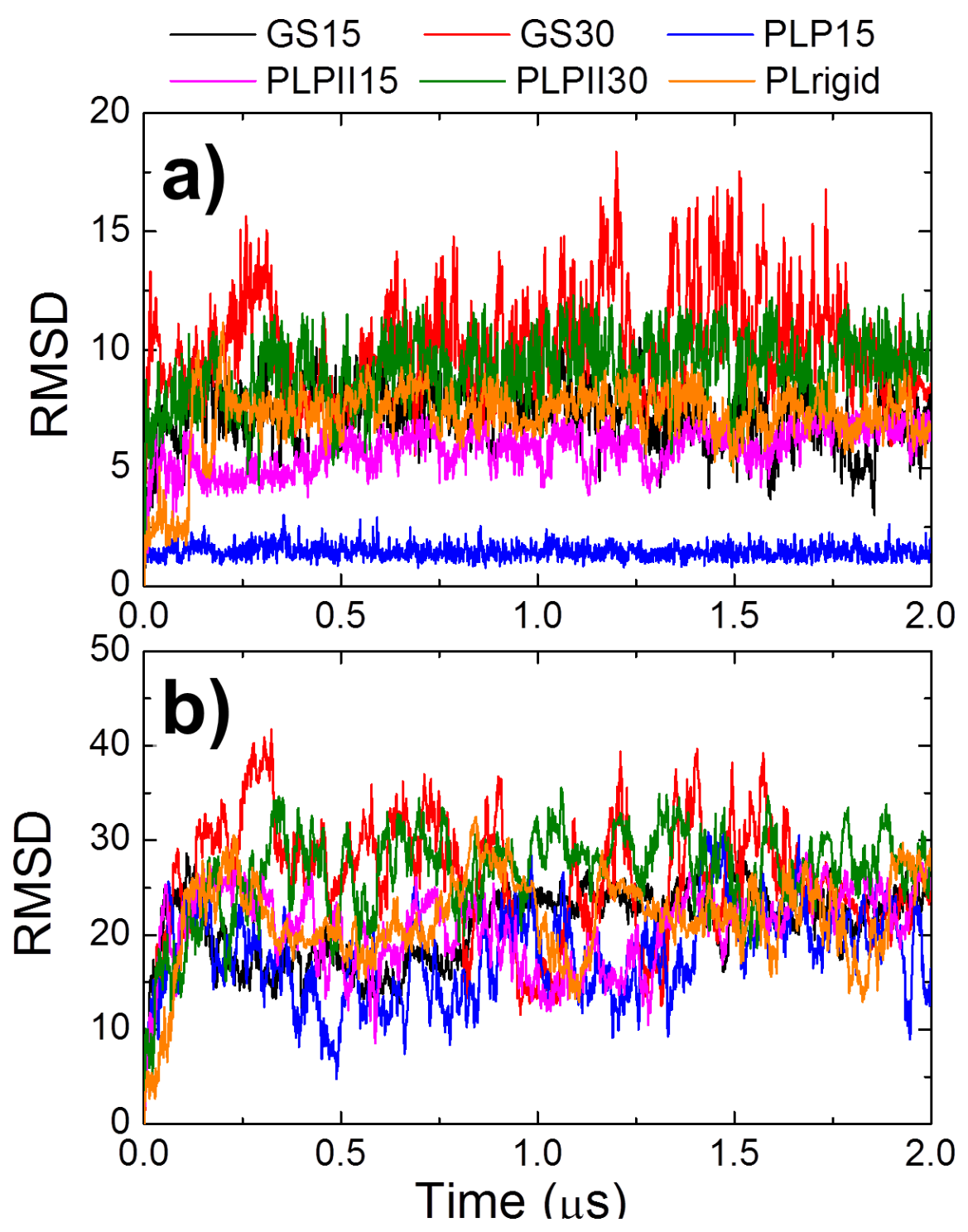




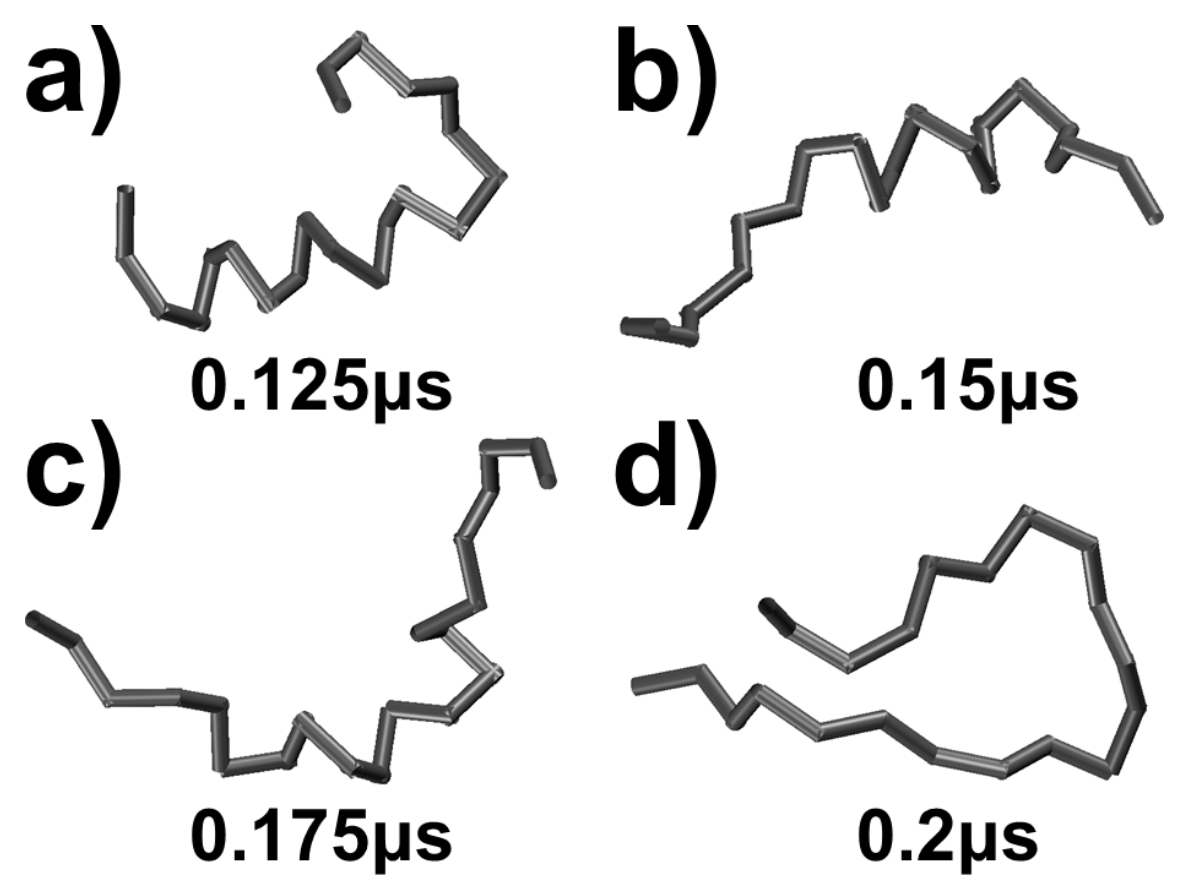

a)

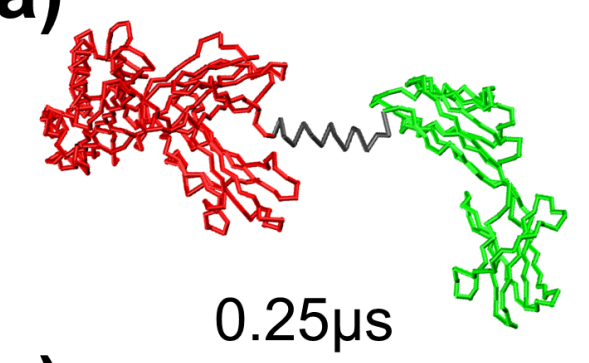

b)

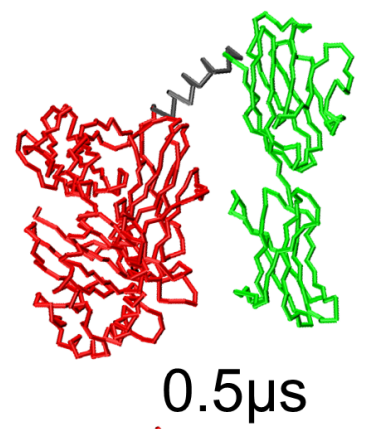

c)

d)

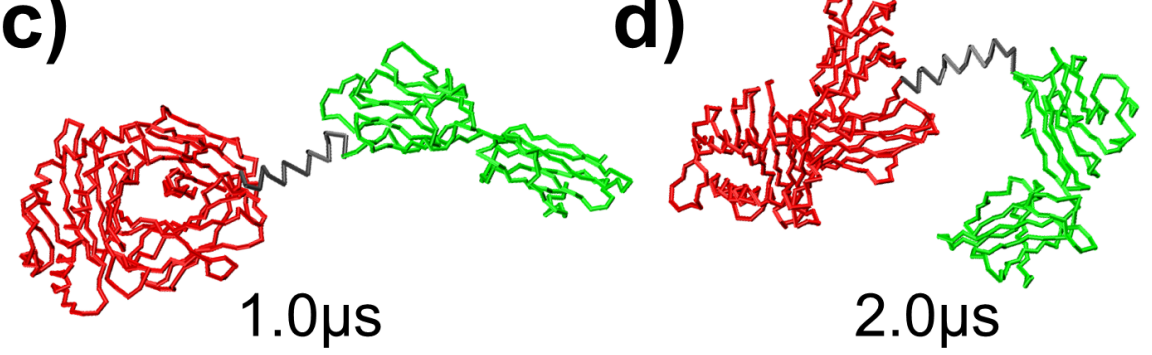



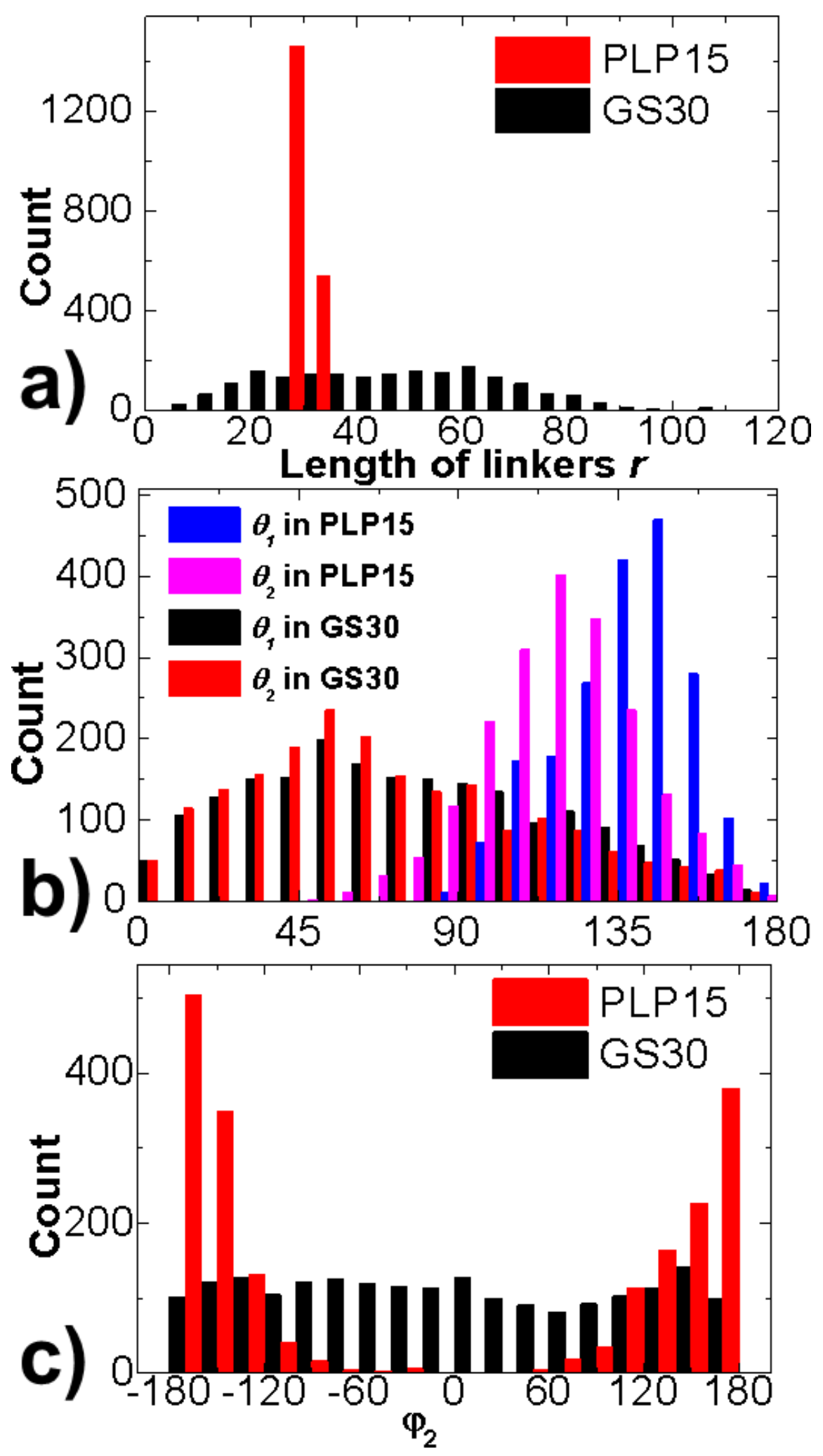

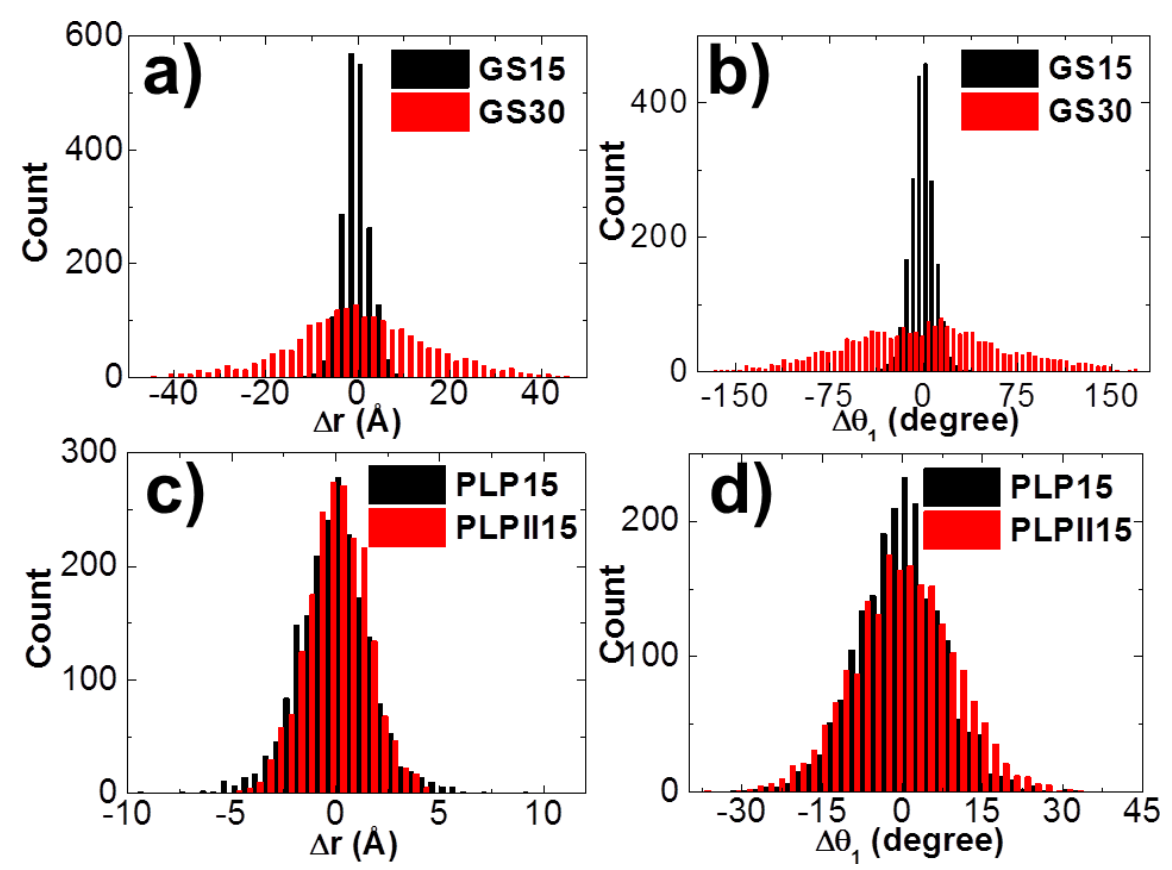

a)
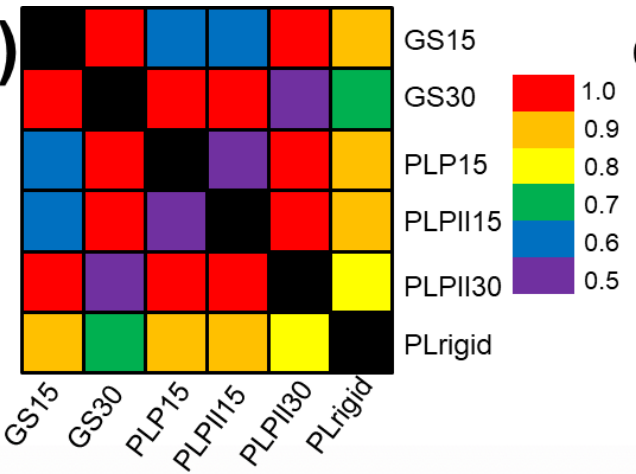

C)
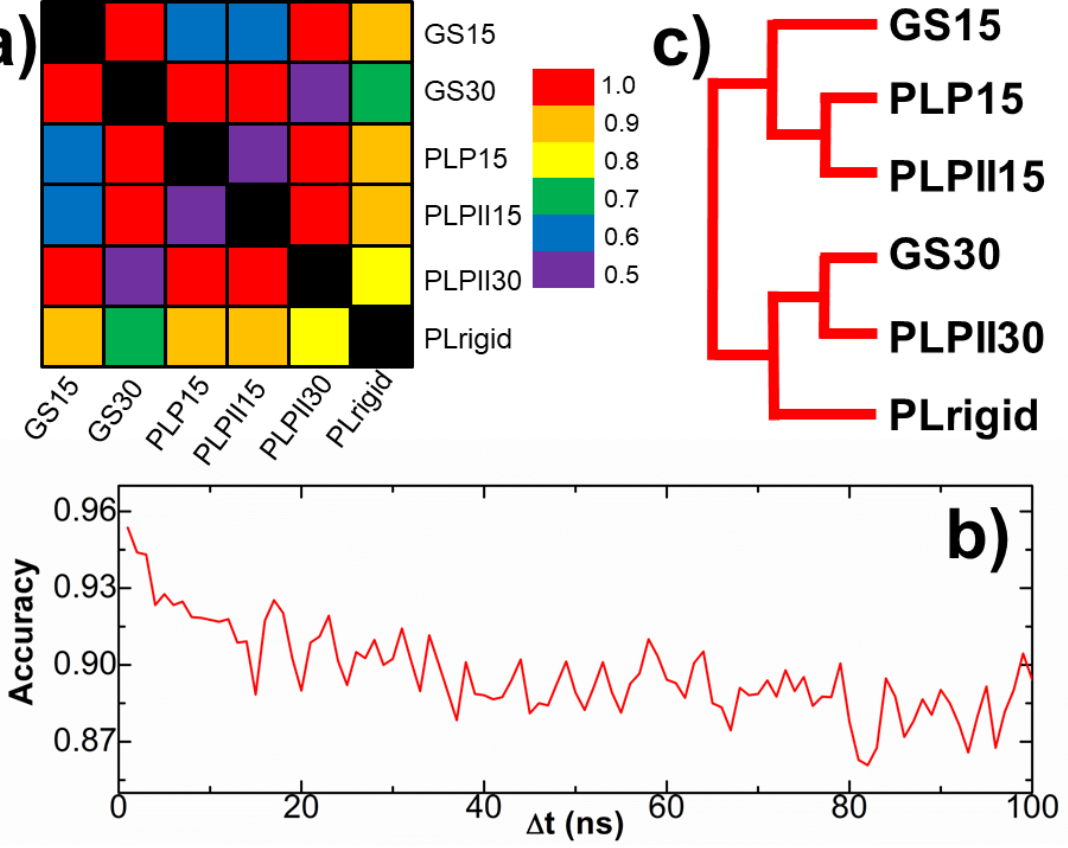